\title{
Fatores de risco e proteção de doenças crônicas em adultos: estudo de base populacional em uma cidade de médio porte no sul do Brasil
}

\author{
Risk and protective factors for chronic diseases in adults: population-based study in a \\ medium-sized town in the South of Brazil \\ Factores de riesgo y protección para enfermedades crónicas en adultos: estudio de base \\ poblacional en una ciudad de tamaño mediano en el sur de Brasil
}

Carine Baumgartel, Mihaela Onofrei, Leo Lynce Valle Lacerda, Luciane Peter Grillo, Tatiana Mezadri

\section{Resumo}

Introdução: As doenças crônicas constituem sério problema de saúde pública, caracterizadas por uma etiologia incerta, múltiplos fatores de risco, longos períodos de latência, curso prolongado, origem não infecciosa e associadas a deficiências e incapacidades funcionais. Objetivos: Determinar a prevalência e a distribuição dos principais fatores de risco e proteção para doenças crônicas não transmissíveis na população adulta, estratificada por sexo e faixa etária no município de Itajaí, SC. Métodos: A amostra foi constituída por 432 adultos, entre julho de 2013 e junho de 2014. Aplicou-se um questionário, composto por fatores de risco e proteção para doenças crônicas não transmissíveis. Resultados: A amostra foi composta por $48,4 \%$ do sexo masculino e $51,6 \%$ do sexo feminino, predominando a faixa etária de 20 a 39 anos $(56,2 \%)$, estado civil casado e/ou união estável $(70,4 \%)$ e com nível de escolaridade de até 11 anos $(46,3 \%)$. Em relação aos fatores de risco, quando comparados com os dados nacional e estadual, os sexos masculino e feminino apresentaram prevalências significativamente superiores, respectivamente, para: tabagismo $(22,5 \% ; 17,0 \%)$; consumo de refrigerantes ou suco artificial $(45,5 \% ; 34,5 \%)$, leite integral $(72,3 \% ; 70,9 \%)$, carne com gordura $(58,4 \% ; 49,3 \%)$, obesidade $(24,2 \% ; 21,3 \%)$ e autoavaliação negativa de saúde $(19,1 \% ; 30,9 \%)$. Quanto aos fatores de proteção verificaram-se valores superiores e significativos para consumo regular de frutas e hortaliças $(51,2 \% ; 70,4 \%)$ e inferiores para o consumo recomendado de frutas e hortaliças $(9,6 \% ; 16,1 \%)$, feijão $(46,9 \% ; 40,4 \%)$ e atividade física $(30,1 \% ; 39,0 \%)$. Conclusão: Os resultados apontaram que as maiores prevalências de fatores de risco estão relacionadas à tríade ingestão alimentar inadequada, inatividade física e tabagismo, destacando-se as elevadas proporções de autoavaliação negativa da saúde.

\begin{abstract}
Introduction: Chronic diseases constitute a serious public health problem, characterized by an uncertain aetiology, multiple risk factors, long periods of latency, prolonged course, non-infectious origin and association with disability and functional disability. Objectives: To determine the prevalence and distribution of major risk factors and protection for chronic diseases in the adult population, stratified by sex and age group in Itajaí, Santa Catarina. Methods: The sample consisted of 432 adults, aged 20-59 years between July/2013 and June/2014. A questionnaire was applied, consisting of risk and protection factors for chronic noncommunicable diseases. Results: The sample comprised $48.4 \%$ men and $51.6 \%$ women with predominant age group $20-39$ years $(56.2 \%)$, being married and/or cohabiting $(70.4 \%)$ and education level of up to eleven years (46.3\%). Regarding risk factors, compared with the national and state data, men and women had significant higher prevalence rates respectively for smoking $(22.5 \% ; 17.0 \%)$; consumption of sodas or artificial juice $(45.5 \% ; 34.5 \%)$, whole milk $(72.3 \% ; 70.9 \%)$, meat with visible fat $(58.4 \% ; 49.3 \%)$, obesity $(24.2 \% ; 21.3 \%)$ and negative self-assessed health $(19.1 \% ; 30.9 \%)$. With regard to protective factors and significant higher values for the regular consumption of fruits and vegetables $(51.2 \% ; 70.4 \%)$ and lower for the recommended consumption of fruits and vegetables $(9.6 \% ; 16.1 \%)$, beans $(46.9 \% ; 40.4 \%)$ and physical activity during leisure time $(30.1 \% ; 39.0 \%)$. Conclusions: The results showed that the highest prevalence of risk factors for chronic diseases is related to triad inadequate food intake, physical inactivity and smoking, specially the high proportions of negative self-rated health.
\end{abstract}

Como citar: Baumgartel C, Onofrei M, Lacerda LLV, Grillo LP, Mezadri T. Fatores de risco e proteção de doenças crônicas em adultos: estudo de base populacional em uma cidade de médio porte no sul do Brasil. Rev Bras Med Fam Comunidade. 2016;11(38):1-13. http://dx.doi.org/10.5712/rbmfc11(38)1248
Palavras-chave:

Doença Crônica

Fatores de Risco

Adulto

Inquéritos Epidemiológicos

Keywords:

Chronic Disease

Risk Factors

Adult

Health Surveys

Fonte de financiamento declaram não haver.

Parecer CEP:

392.197/13 (Univali), aprovado

25/10/2011.

Conflito de interesses:

declaram não haver.

Procedência e revisão por pares: revisado por pares.

Recebido em: 26/11/2015.

Aprovado em: 09/09/2016. 


\section{Resumen}

Introducción: Las enfermedades crónicas constituyen un problema grave de salud pública, caracterizado por una etiología incierta, múltiples factores de riesgo, largos períodos de latencia, curso prolongado, origen no infeccioso y asociación con deficiencias y discapacidades funcionales. Objetivos: Determinar la prevalencia y la distribución de los principales factores de riesgo y protección para enfermedades crónicas no transmisibles en la población adulta, estratificada por sexo y grupo de edad, en la ciudad de Itajaí, Santa Catarina. Métodos: La muestra consistió en 432 adultos entre julio de 2013 y junio de 2014. Se aplicó un cuestionario, que consta de factores de riesgo y protección para enfermedades crónicas. Resultados: La muestra estuvo constituida por $48,4 \%$ hombres y $51,6 \%$ mujeres, rango de edad predominante fue de $20-39$ años $(56,2 \%)$, que se casaron y/o unión de hecho $(70,4 \%)$ y el nivel de educación de hasta once años (46,3\%). En cuanto a los factores de riesgo, en comparación con los datos nacionales y estatales, hombres y mujeres mostraron significativa prevalencia más alta, respectivamente, para: fumadores $(22,5 \%$; $17,0 \%)$; consumo de bebidas gaseosas o jugos artificiales $(45,5 \% ; 34,5 \%)$, leche entera $(72,3 \% ; 70,9 \%)$, carne con grasa $(58,4 \% ; 49,3 \%)$, obesidad $(24,2 \% ; 21,3 \%)$ y la autoevaluación de salud negativa $(19,1 \% ; 30,9 \%)$. En cuanto a los factores de protección que se consideren valores superiores y significativos para el consumo regular de frutas y verduras $(51,2 \% ; 70,4 \%)$ y más baja para la ingesta recomendada de frutas y verduras $(9,6 \% ; 16,1 \%)$, frijoles $(46,9 \% ; 40,4 \%)$ y actividad física $(30,1 \% ; 39,0 \%)$. Conclusión: Los resultados mostraron que la mayor prevalencia de factores de riesgo está relacionada con la tríada - ingesta inadecuada de alimentos, inactividad física y tabaquismo, destacando las proporciones altas de autoevaluación negativa de salud.
Palabras clave:

Enfermedad Crónica

Factores de Riesgo

Adulto

Encuestas de Salud

\section{Introdução}

As doenças crônicas não transmissíveis (DCNT) constituem sério problema de saúde pública, tanto nos países ricos quanto nos de média e baixa renda. ${ }^{1,2}$ Caracterizam-se por ter uma etiologia incerta, múltiplos fatores de risco, longos períodos de latência, curso prolongado, origem não infecciosa e por estarem associadas a deficiências e incapacidades funcionais, ${ }^{3}$ sendo responsáveis por $63 \%$ das mortes em todo o mundo, segundo estimativas da Organização Mundial da Saúde ${ }^{4}$ além de resultarem na necessidade de tratamentos contínuos que, consequentemente, sobrecarregam o Sistema Único de Saúde (SUS) com consultas recorrentes e custos elevados de medicamentos para controle. ${ }^{5}$

Mais de $70 \%$ das mortes no Brasil foram atribuídas às DCNT (doenças cardiovasculares, doenças respiratórias crônicas, diabetes, câncer e outras). Crescimento da renda, industrialização e mecanização da produção, urbanização, maior acesso a alimentos em geral, incluindo os processados, e globalização de hábitos não saudáveis produziram rápida transição nutricional, expondo a população cada vez mais ao risco destas doenças. ${ }^{6,7}$

Os fatores de risco indicados para monitoramento são aqueles que mais repercutem na morbidade e mortalidade para doenças crônicas, que podem ser modificados mediante intervenção no âmbito da atenção à saúde e que são relativamente simples de serem medidos, tais como: consumo de tabaco e álcool, excesso de peso, inatividade física e alimentação não saudável. ${ }^{8}$

Integrante da atenção básica à saúde, a Saúde da Família é uma estratégia de mudança do Sistema Único de Saúde de um modelo meramente assistencial e hospitalocêntrico para um baseado na atenção básica como sua porta de entrada. Mais abrangente do que o modelo tradicional, a Saúde da Família engloba ações de promoção da saúde, prevenção de doenças, assistência aos agravos mais prevalentes, recuperação e reabilitação. Por essas características, a Estratégia Saúde da Família (ESF) tem um grande potencial de se integrar à rede de vigilância em saúde voltada para as doenças crônicas. ${ }^{9}$

Desde a década de 1970, estratégias de controle dos fatores de risco dessas doenças, combinadas com educação comunitária e monitoramento dos indivíduos de alto risco, contribuíram para uma queda substancial na mortalidade em quase todos os países desenvolvidos. ${ }^{10}$ 
O Plano de Ações Estratégicas para o Enfrentamento das Doenças Crônicas Não Transmissíveis, 2011-2022 define e prioriza as ações de investimentos necessárias à preparação do país para enfrentar e deter as DCNT e seus fatores de risco nos próximos dez anos. O documento aborda os quatro principais grupos de doenças (cardiovasculares, câncer, respiratórias crônicas e diabetes) e seus fatores de risco em comum modificáveis (tabagismo, álcool, inatividade física, alimentação inadequada e obesidade) e define três eixos de atuação: I. Vigilância, informação, avaliação e monitoramento; II. Promoção da saúde; e III. Cuidado Integral de Doenças Crônicas não Transmissíveis. ${ }^{4}$

São escassos os trabalhos existentes sobre a vigilância de fatores de risco e proteção para DCNT em municípios de médio porte. As grandes cidades perderam o posto de principais polos de atração populacional para os municípios de médio porte com população entre 100 mil e 500 mil habitantes. Incentivados pelo processo de descentralização da indústria e pela expansão do agronegócio, as cidades de 100 mil a 500 mil habitantes aumentaram de $23,3 \%$ para $25,5 \%$ a participação na população brasileira. ${ }^{11}$

Dessa forma, faz-se necessário o conhecimento das prevalências e distribuições de fatores de risco para doenças crônicas nos municípios brasileiros, com o compromisso de investigar e intervir no âmbito das realidades regionais de forma a auxiliar no monitoramento de ações de redução e controle destes fatores.

A escolha do tema atende ao primeiro eixo de atuação do Plano de Ações Estratégicas para o Enfrentamento das Doenças Crônicas Não Transmissíveis, 2011-2022 que fomenta e apoia o desenvolvimento e o fortalecimento da vigilância integrada de DCNT e seus fatores de proteção e risco modificáveis, com ênfase nos inquéritos nacionais e locais. ${ }^{12} \mathrm{O}$ tema proposto também se deve a tendências desfavoráveis na maioria dos principais fatores de risco, constatando a importância de diagnósticos locais amplos e atualizados que deem subsídios ao planejamento de ações de promoção da saúde e prevenção de DCNT de acordo com as especificidades do grupo avaliado.

O objetivo deste trabalho foi determinar a prevalência e distribuição dos principais fatores de risco e proteção para doenças crônicas não transmissíveis na população adulta (referência da Organização Mundial da Saúde - OMS), estratificada por sexo e faixa etária, do município de Itajaí, SC, comparando os resultados com aqueles obtidos pela pesquisa via inquérito telefônico (VIGITEL) para o Brasil e, especificamente, Florianópolis, SC, de forma a obter uma caracterização do município em relação ao âmbito estadual e nacional.

\section{Métodos}

\section{Desenho, população do estudo e amostragem}

O presente estudo foi caracterizado como transversal de base populacional, desenvolvido nas equipes de Estratégia Saúde da Família do município de Itajaí. A cidade de Itajaí está localizada no litoral norte do estado de Santa Catarina a $93 \mathrm{Km}$ de Florianópolis, capital do Estado. Atualmente, possui 183.373 mil habitantes e um contingente de 110.013 adultos. ${ }^{11}$

A população estudada foi constituída por 110.013 pessoas de ambos os sexos, com idades compreendidas entre 20 e 59 anos. A amostra foi inicialmente representada por 440 indivíduos com idade igual ou superior a 20 anos, garantindo os estimadores dos parâmetros estudados a um erro máximo de 
$5 \%$, com um grau de confiança de $95 \%$ além de margem de perda acima de $10 \%$. Considerando-se que no período da coleta a cobertura da atenção básica no município era de $100 \%$, utilizou-se a distribuição das unidades básicas de saúde como marco amostral, e como unidades amostrais os registros de pessoas nas unidades de saúde.

O sorteio dos indivíduos foi realizado por meio de códigos atribuídos às fichas cadastrais, com a utilização da função de número aleatório disponível no MS Excel. A amostra foi estratificada proporcionalmente por sexo e faixa etária com ajuste por conglomerado, proporcionalmente ao número de pessoas cadastradas por unidade de saúde. Todas as unidades de saúde do município participaram da amostragem, assegurando-se a representatividade geográfica. Estas foram utilizadas como pontos facilitadores de coleta de dados com as entrevistas nas salas de espera anteriomente à consulta. No entanto, para a amostra masculina, foram realizadas visitas domiciliares, tendo em vista a baixa procura deste estrato nas unidades de saúde. Os critérios de inclusão foram: idade entre 20 e 59 anos. O critério de exclusão foi: ser gestante ou nutriz.

Do sorteio inicial dos 440 indivíduos, 27 não puderam ser contatados por incorreção ou desatualização do cadastro. Dos 413 indivíduos, foram recolhidos dados de 397. Dos demais, dez não foram em encontrados e seis não assinaram o Termo de Consentimento Livre e Esclarecido (TCLE). Uma reamostragem de 43 indivíduos foi realizada com novo sorteio de acordo com as unidades de saúde, sexo e faixa etária. Nesta segunda etapa foram contatados 40 indivíduos e destes recolhidos dados de 38, pois dois não aceitaram assinar o TCLE. Ao final da coleta foram descartados dados de três indivíduos devido a inconsistências de coleta, totalizando 432 indivíduos, ou seja, uma amostra 12,6\% acima do tamanho mínimo amostral.

\section{Coleta de dados e definição de variáveis}

Os dados foram coletados mediante aplicação de um questionário estruturado fechado de múltipla escolha por alunos supervisionados e previamente capacitados para tal fim. Um estudo piloto foi realizado na unidade de maior número de atendimentos para testar o instrumento nas três faixas etárias: 20-39 anos, 40-49 anos e 50-59 anos.

O instrumento de coleta de dados foi um questionário composto por indicadores monitorados pelo Sistema de Vigilância de Fatores de Risco e Proteção para Doenças Crônicas por Inquérito Telefônico. ${ }^{13}$

Dentre os fatores de risco, as seguintes variáveis foram avaliadas: tabagismo, presença de excesso de peso e obesidade, hábito de consumo de refrigerante e suco artificial e de carnes e leite com excesso de gordura, consumo abusivo de bebidas alcoólicas, autoavaliação de estado de saúde ruim, referência a diagnóstico médico de hipertensão arterial, diabetes, dislipidemias. Os fatores de proteção avaliados foram: hábito de consumo regular e recomendado de frutas, legumes, verduras e feijão e atividade física suficiente no tempo livre e/ou deslocamento. A definição desses indicadores é apresentada a seguir:

Foram caracterizados como tabagistas os usuários que mantêm o hábito de fumar cigarros, independentemente da quantidade. Para o excesso de peso, foi considerado o indivíduo com índice de massa corporal maior ou igual $25 \mathrm{~kg} / \mathrm{m},{ }^{14}$ calculado a partir do peso em quilos dividido pelo quadrado da altura, ambos autorreferidos, e para obesidade o indivíduo com índice de massa corporal maior ou igual $30 \mathrm{~kg} / \mathrm{m} .{ }^{14}$ 
Avaliou-se o percentual de adultos que consomem frutas e hortaliças regularmente: consumo em cinco ou mais dias da semana, percentual de adultos que consomem frutas e hortaliças conforme recomendado: consumo de cinco ou mais vezes por dia em cinco ou mais dias da semana. Para o cálculo de frutas e suco de frutas, foi considerada cada fruta ou cada suco de fruta como equivalente a uma porção, limitando-se em três o número máximo de porções diárias computado para frutas e em um o número máximo computado para sucos. No caso de hortaliças, computou-se um número máximo de quatro porções diárias. Também foi verificado o percentual de adultos que costumam consumir carnes com gordura visível, leite com teor integral de gordura, refrigerante ou suco artificial cinco ou mais dias por semana e feijão cinco ou mais dias por semana.

$\mathrm{Na}$ questão da atividade física foi considerado o percentual de adultos que praticavam atividades físicas no tempo livre e/ou deslocamento equivalentes a, pelo menos, 150 minutos de atividade física de intensidade moderada por semana, além do percentual de adultos que despendem três ou mais horas diárias vendo televisão.

Foram avaliados o percentual de adultos que consumiram bebidas alcoólicas de forma abusiva. Foi considerado consumo abusivo de bebidas alcoólicas cinco ou mais doses (homem) ou quatro ou mais doses (mulher) em uma única ocasião, pelo menos uma vez nos últimos 30 dias. Uma dose de bebida alcoólica corresponde a uma lata de cerveja, uma taça de vinho ou uma dose de cachaça, whisky ou qualquer outra bebida alcoólica destilada.

Para a autoavaliação do estado de saúde, foram avaliados o percentual de adultos que autoavaliaram o estado de saúde como ruim ou muito ruim e o percentual de adultos que referiram diagnóstico médico de hipertensão arterial, diabetes e dislipidemia.

Também foram coletadas informações complementares sobre o nível de escolaridade ( 0 a 8, 9 a 11 ou $\geq 12$ anos de estudo), idade e estado civil (casado, solteiro).

Os resultados obtidos neste estudo foram comparadas com os dados nacional (Brasil) e estadual (Florianópolis) do Sistema de Vigilância de Fatores de Risco e Proteção para Doenças Crônicas por Inquérito Telefônico de 2013. ${ }^{13}$

\section{Aspectos Éticos}

O projeto de pesquisa foi aprovado pela Comissão de Ética em Pesquisa da Universidade do Vale do Itajaí sob protocolo no 392.197/13. Todos os participantes assinaram o TCLE e receberam orientações de promoção e educação em saúde ao término da entrevista. A partir dos resultados obtidos foram desenvolvidos materiais educativos para serem utilizados nas Unidades Básicas de Saúde.

\section{Análise dos dados}

Foram calculadas as proporções, razões de prevalência e seus respectivos intervalos com 95\% de confiança dos fatores de risco e de proteção para as doenças crônicas para a amostra geral e de acordo com o sexo. Os dados foram analisados por meio do STATISTICA v. 10, utilizando-se o teste da diferença entre proporções para a comparação das amostras. O nível de significância adotado foi de $5 \%$. 


\section{Resultados}

Participaram do estudo 432 indivíduos residentes em Itajaí, SC, sendo $209(48,4 \%)$ do sexo masculino e $223(51,6 \%)$ do sexo feminino. A Tabela 1 apresenta as características dos usuários avaliados. Observa-se predominância da faixa etária de 20 a 39 anos, estado civil casado/união estável e com nível de escolaridade de 9 a 11 anos.

Tabela 1. Características dos usuários das Unidades Básicas de Saúde de Itajaí, SC, 2014.

\begin{tabular}{lccc} 
& & & Percentual \\
\cline { 3 - 4 } Variável & $\mathbf{n}$ & Masculino & Feminino \\
\hline Amostra & 432 & 48,38 & 51,62 \\
\hline Idade & & & 60,54 \\
\hline $20-39$ & 243 & 51,67 & 17,04 \\
$40-49$ & 85 & 22,49 & 22,42 \\
$50-59$ & 104 & 25,84 & \\
\hline Estado civil & & & 31,84 \\
\hline Solteiro & 128 & 27,27 & 68,16 \\
Casado/união estável & 304 & 72,73 & 42,60 \\
\hline Escolaridade & & & 46,64 \\
\hline 0 a 8 anos & 187 & 44,02 & 10,76 \\
9 a 11 anos & 200 & 45,93 & 10,05 \\
12 anos ou mais & 45 & &
\end{tabular}

As prevalências e intervalos de confiança dos fatores de risco e proteção para doenças crônicas estão descritos na Tabela 2. Quando se compara os sexos, verificam-se prevalências significativamente superiores para os homens com relação ao tabagismo, consumo de refrigerantes e/ou suco artificial e de carne com gordura visível, excesso de peso, assistir televisão por mais de três horas e consumo abusivo de bebida alcoólica e para as mulheres a autoavaliação negativa do estado de saúde e o consumo regular e recomendado de frutas e hortaliças.

A Tabela 3 descreve as médias e intervalos de confiança dos fatores de risco e proteção para DCNT no sexo feminino comparando com os dados de Florianópolis e do Brasil, provindos da pesquisa VIGITEL 2013. ${ }^{13}$ Observados os fatores de risco, quando se compara a amostra feminina do estudo com o dado estadual, verificam-se prevalências superiores e significativas para as variáveis: tabagismo, consumo de refrigerante e/ou suco artificial, consumo de leite com teor integral de gordura e de carnes com gordura visível, obesidade, autoavaliação negativa do estado de saúde, hipertensão e diabetes autorreferidas e prevalência inferior somente para o excesso de peso.

Ao comparar estas mulheres com dados em nível nacional, constatam-se prevalências superiores para as variáveis: tabagismo, consumo de refrigerante ou suco artificial, leite com teor integral de gordura e carnes com gordura visível, autoavaliação negativa do estado de saúde e foi encontrada prevalência inferior para excesso de peso e dislipidemia autorreferida.

Em relação aos fatores de proteção, a amostra feminina apresentou prevalência superior com relação ao dado nacional para a variável consumo regular de frutas e hortaliças e prevalências significativas inferiores para o consumo recomendado de frutas, hortaliças e feijão e prática de atividade 
Tabela 2. Prevalências e respectivos intervalos com 95\% de confiança (IC 95\%) dos fatores de risco e proteção para doenças crônicas não transmissíveis, segundo o sexo. Itajaí, SC, 2014.

\begin{tabular}{|c|c|c|c|}
\hline \multirow[b]{2}{*}{ Fator } & \multicolumn{2}{|c|}{ Prevalência } & \multirow[b]{2}{*}{$\mathbf{p}^{*}$} \\
\hline & $\begin{array}{l}\text { Homens } \\
\% \text { (IC95\%) }\end{array}$ & $\begin{array}{l}\text { Mulheres } \\
\% \text { (IC95\%) }\end{array}$ & \\
\hline Tabagismo & $22,49(16,83-28,15)$ & $17,04(12,11-21,98)$ & $<0,001$ \\
\hline Consumo de refrigerante/suco artificial & $45,45(38,70-52,21)$ & $34,53(28,29-40,77)$ & 0,018 \\
\hline Consumo de carnes com gordura & $48,33(41,55-55,10)$ & $34,98(28,72-41,24)$ & 0,005 \\
\hline Excesso de peso (IMC $\left.\geq 25 \mathrm{~kg} / \mathrm{m}^{2}\right)$ & $34,30(27,83-40,77)$ & $26,54(20,58-32,50)$ & $<0,001$ \\
\hline Obesidade $\left(\mathrm{IMC} \geq 30 \mathrm{~kg} / \mathrm{m}^{2}\right)$ & $24,15(18,32-29,99)$ & $21,33(15,80-26,85)$ & 0,492 \\
\hline Assistir TV por mais de três horas & $43,06(36,35-49,45)$ & $25,56(19,84-31,29)$ & 0,001 \\
\hline Consumo abusivo de bebida & $30,62(24,37-36,87)$ & $13,00(8,59-17,42)$ & $<0,001$ \\
\hline Dislipidemia autorreferida & $20,57(15,09-26,05)$ & $16,56(11,71-21,47)$ & 0,283 \\
\hline \multicolumn{4}{|l|}{ Fator de proteção } \\
\hline Consumo regular de frutas/hortaliças & $51,20(44,42-57,97)$ & $70,40(64,41-76,39)$ & $<0,001$ \\
\hline Consumo recomendado de frutas/hortaliças & $9,57(5,58-13,56)$ & $16,14(11.31-20,97)$ & 0,042 \\
\hline Consumo de feijão & $46,89(40,12-53,66)$ & $40,36(33,92-46,80)$ & 0,171 \\
\hline Prática de atividade física & $30,14(23,92-36,36)$ & $39,01(32,61-45,42)$ & 0,098 \\
\hline
\end{tabular}

* teste de proporções ao nível de $5 \%$.

Tabela 3. Média e intervalo de confiança dos fatores de risco e proteção: dados Brasil, Florianópolis e Itajaí, amostra feminina, 2014.

\begin{tabular}{|c|c|c|c|c|c|}
\hline Fator & Brasil (1) & Florianópolis (2) & Amostra & $p(1)$ & $\mathrm{p}(2)$ \\
\hline \multicolumn{6}{|l|}{ Fator de risco } \\
\hline Tabagismo & $8,6(7,9-9,3)$ & $10,6(8,4-12,8)$ & $17,0(12,1-22,0)$ & $<0,001$ & 0,006 \\
\hline Consumo de refrigerante/suco artificial & $20,4(19,4-21,3)$ & $16,3(13,6-19,0)$ & $34,5(28,3-40,8)$ & $<0,001$ & $<0,001$ \\
\hline Consumo de leite integral & $50,9(49,8-52,0)$ & $42,5(38,8-46,1)$ & $70,9(64,9-76,8)$ & $<0,001$ & $<0,001$ \\
\hline Consumo de carnes com gordura & $22,2(21,2-23,1)$ & $15,8(13,1-18,6)$ & $49,3(42,8-55,9)$ & $<0,001$ & $<0,001$ \\
\hline Excesso de peso (IMC $\left.\geq 25 \mathrm{~kg} / \mathrm{m}^{2}\right)$ & $47,4(46,3-48,5)$ & $41,4(37,9-45,0)$ & $26,5(20,6-32,5)$ & $<0,001$ & $<0,001$ \\
\hline Obesidade $\left(\mathrm{IMC} \geq 30 \mathrm{~kg} / \mathrm{m}^{2}\right)$ & $17,5(16,7-18,4)$ & $14,6(12,2-17,0)$ & $21,3(15,8-26,9)$ & 0,137 & 0,013 \\
\hline Assistir TV por mais de três horas & $29,0(28,0-30,0)$ & $22,5(19,7-25,4)$ & $25,6(19,8-31,3)$ & 0,265 & 0,312 \\
\hline Consumo abusivo de bebida alcoólica & $9,7(9,0-10,4)$ & $10,9(8,5-13,4)$ & $13,0(8,6-17,4)$ & 0,097 & 0,361 \\
\hline Autoavaliação negativa de saúde & $5,8(5,3-6,3)$ & $6,4(4,7-8,2)$ & $30,9(24,0-36,1)$ & $<0,001$ & $<0,001$ \\
\hline Hipertensão autorreferida & $26,3(25,4-27,3)$ & $21,5(18,9-24,1)$ & $30,0(24,0-36,1)$ & 0,211 & 0,005 \\
\hline Diabetes autorreferido & $7,2(6,7-7,7)$ & $6,1(4,7-7,5)$ & $9,87(5,9-13,8)$ & 0,125 & 0,038 \\
\hline Dislipidemia autorreferida & $22,9(22,1-23,8)$ & $20,7(18,0-23,5)$ & $16,6(11,7-21,5)$ & 0,026 & 0,160 \\
\hline \multicolumn{6}{|l|}{ Fator de proteção } \\
\hline Consumo regular de frutas/hortaliças & $41,5(40,4-42,5)$ & $55,8(52,1-59,4)$ & $70,4(64,4-76,4)$ & $<0,001$ & $<0,001$ \\
\hline Consumo recomendado de frutas/hortaliças & $27,3(26,3-28,2)$ & $35,6(32,2-39,0)$ & $16,1(11,3-21)$ & 0,002 & $<0,001$ \\
\hline Consumo de feijão & $61,7(60,6-62,7)$ & $33,8(30,4-37,3)$ & $40,4(33,9-46,8)$ & $<0,001$ & 0,057 \\
\hline Prática de atividade física & $57,4(56,3-58,5)$ & $51,5(47,9-55,1)$ & $39,0(32,6-45,4)$ & $<0,001$ & 0,006 \\
\hline
\end{tabular}

Nota: valores estaduais e nacionais conforme pesquisa VIGITEL 2013 (Brasil, Ministério da Saúde 2014). ${ }^{13}$ Os valores de p se referem a significância da diferença entre as proporções da amostra e do Brasil (1) e Florianópolis (2), respectivamente. IMC=índice de massa corporal. 
física. Os resultados foram semelhantes quando comparados com o resultado estadual, com exceção para o consumo de feijão.

A Tabela 4 apresenta as médias e intervalos de confiança dos fatores de risco e proteção para DCNT no sexo masculino comparando com os dados estadual (Florianópolis) e nacional (Brasil).

Tabela 4. Média e intervalo de confiança dos fatores de risco e proteção: dados Brasil, Florianópolis e Itajaí, amostra masculina, 2014

\begin{tabular}{|c|c|c|c|c|c|}
\hline Fator & Brasil (1) & Florianópolis (2) & Amostra & $p(1)$ & p (2) \\
\hline \multicolumn{6}{|l|}{ Fator de risco } \\
\hline Tabagismo & $14,4(13,3-15,5)$ & $14,5(11,1-17,9)$ & $22,5(16,8-28,2)$ & $<0,001$ & 0,006 \\
\hline Consumo de refrigerante/suco artificial & $26,7(25,4-28,0)$ & $25,1(21,0-29,3)$ & $45,5(38,7-52,2)$ & $<0,001$ & $<0,001$ \\
\hline Consumo de leite integral & $56,6(55,2-57,9)$ & $50,4(45,8-55,0)$ & $72,3(66,2-78,3)$ & $<0,001$ & $<0,001$ \\
\hline Consumo de carnes com gordura & $41,2(39,9-42,6)$ & $35,4(30,9-39,9)$ & $58,4(51,7-65,1)$ & $<0,001$ & $<0,001$ \\
\hline Excesso de peso(IMC $\left.\geq 25 \mathrm{~kg} / \mathrm{m}^{2}\right)$ & $54,7(53,4-56,1)$ & $56,5(52,0-61,1)$ & $34,3(27,8-40,8)$ & $<0,001$ & $<0,001$ \\
\hline Obesidade(IMC $\left.\geq 30 \mathrm{~kg} / \mathrm{m}^{2}\right)$ & $17,5(16,5-18,5)$ & $16,4(13,2-19,7)$ & $24,2(18,3-30,0)$ & 0,011 & $<0,001$ \\
\hline Assistir TV por mais de três horas & $28,1(26,8-29,3)$ & $27,3(23,2-31,5)$ & $43,1(36,4-49,5)$ & $<0,001$ & $<0,001$ \\
\hline Consumo abusivo de bebida alcoólica & $24,2(23,0-25,4)$ & $26,4(22,2-30,7)$ & $30,6(24,4-36,9)$ & 0,032 & 0,229 \\
\hline Autoavaliação negativa de saúde & $3,8(3,2-4,3)$ & $3,2(1,7-4,7)$ & $19,1(6,8-15,3)$ & $<0,001$ & $<0,001$ \\
\hline Hipertensão autorreferida & $21,5(20,4-22,5)$ & $19,4(16,3-22,5)$ & $28,2(22,1-34,3)$ & 0,019 & 0,006 \\
\hline Diabetes autorreferido & $6,5(5,8-7,2)$ & $4,9(3,5-6,3)$ & $11,0(6,8-15,3)$ & 0,009 & 0,001 \\
\hline Dislipidemia autorreferida & $17,2(16,2-18,2)$ & $16,5(13,5-19,5)$ & $20,6(15,1-26,1)$ & 0,195 & 0,167 \\
\hline \multicolumn{6}{|l|}{ Fator de proteção } \\
\hline Consumo regular de frutas/hortaliças & $29,6(28,4-30,8)$ & $38,4(34,1-42,8)$ & $51,20(44,4-58)$ & $<0,001$ & $<0,001$ \\
\hline Consumo recomendado de frutas/hortaliças & $19,3(18,2-20,4)$ & $23,4(19,6-27,1)$ & $9,6(5,6-13,6)$ & 0,004 & $<0,001$ \\
\hline Consumo de feijão & $73,0(71,8-74,2)$ & $47,2(42,6-51,8)$ & $46,9(40,1-53,7)$ & $<0,001$ & 0,939 \\
\hline Prática de atividade física & $39,9(38,6-41,3)$ & $33,1(29,0-37,3)$ & $30,1(23,9-36,4)$ & 0,004 & 0,413 \\
\hline
\end{tabular}

Nota: valores estaduais e nacionais conforme pesquisa VIGITEL 2013 (Brasil, Ministério da Saúde 2014). ${ }^{13}$ Os valores de $p$ se referem a significância da diferença entre as proporções da amostra e do Brasil (1) e Florianópólis (2) respectivamente. IMC=índice de massa corporal.

Com relação aos fatores de risco, quando se compara a amostra masculina do estudo com os dados nacional e estadual, verificam-se prevalências superiores para as variáveis: tabagismo, consumo de refrigerante ou suco artificial, leite com teor integral de gordura e carnes com gordura visível, obesidade, assistir TV por mais de três horas, autoavaliação negativa do estado de saúde e prevalência inferior para o excesso de peso. O consumo excessivo de bebida alcoólica foi significativamente superior quando comparado com o dado nacional, mas não quando comparados com o estadual.

Para os fatores de proteção, verifica-se prevalência significativamente superior com relação aos dados nacional para a variável consumo regular de frutas e hortaliças e prevalências inferiores para consumo recomendado de frutas e hortaliças, de feijão e prática de atividade física. Ao comparar estes resultados com o dado estadual, verifica-se prevalência superior para a variável consumo regular de frutas e hortaliças e prevalência inferior para o consumo recomendado.

\section{Discussão}

Com base na amostra estudada, percebe-se uma junção de fatores que possibilitam risco considerável ao desenvolvimento de doenças crônicas não transmissíveis em ambos os sexos. 
Os homens e mulheres avaliados apresentaram prevalências significativas elevadas quando comparados aos dados nacional e estadual com relação ao tabagismo. Este é um importante fator de risco para o desenvolvimento de uma série de doenças crônicas, tais como câncer, doenças pulmonares e doenças cardiovasculares, de modo que o uso do tabaco continua sendo líder global entre as causas de mortes evitáveis. ${ }^{15}$

O Global Adult Tobacco Survey é uma pesquisa de monitoramento sistemático do uso do tabaco e já foi implementada em 14 países, favorecendo a produção de informação abrangente e padronizada com representatividade nacional e comparabilidade internacional. O resultado mostrou que existiam no Brasil, em 2008, 24,6 milhões de fumantes nessa população adulta (17,2\%), com uma prevalência entre homens de $21,6 \%$ e entre as mulheres de $13,1 \%$. Outra constatação foi que os moradores da região Sul apresentaram maiores prevalências comparados com os dados nacionais (19\%). ${ }^{16}$

O Sistema de Vigilância de Fatores de Risco e Proteção para Doenças Crônicas por Inquérito Telefônico utiliza indicadores do consumo de alimentos considerados marcadores de padrões saudáveis e não saudáveis de alimentação. No primeiro caso, avalia-se a frequência de consumo de frutas, hortaliças (legumes e verduras) e feijão. No segundo caso, avalia-se o hábito de consumir carnes com excesso de gordura (sem remover a gordura visível) e de consumir leite com teor integral de gordura, além do consumo de refrigerantes e sucos artificiais. ${ }^{13}$

Em outro estudo realizado no estado de Santa Catarina, os pesquisadores encontraram prevalências superiores para mulheres e homens com relação ao consumo de frutas/hortaliças e de feijão, respectivamente, ao contrário do constatado no presente trabalho. ${ }^{17} \mathrm{~A}$ OMS recomenda a ingestão diária de pelo menos 400 gramas de frutas e hortaliças, ${ }^{18}$ o que equivale, aproximadamente, ao consumo diário de cinco porções desses alimentos.

O Guia Alimentar para a População Brasileira ${ }^{19}$ orienta a ingestão de pelo menos uma porção diária de feijão ou outra leguminosa (ervilha seca, grão-de-bico, lentilha, soja), pelo alto teor em fibras encontrado nesses alimentos, além de sua relativa baixa densidade energética (uma porção de feijão corresponde a aproximadamente $5 \%$ das calorias diárias), desde que evitadas preparações com alto teor de gordura. 0 consumo de feijão foi significativamente baixo tanto para homens quanto para mulheres quando comparado com os dados nacionais.

Aliada à alimentação inadequada, a falta de atividade física pode provocar a obesidade e a hipertensão, por exemplo, e ainda pode desencadear a hiperglicemia e aumento dos níveis de colesterol no sangue. Esses fatores, por sua vez, contribuem para o desenvolvimento de mais doenças crônicas, como a doença isquêmica do coração, diabetes e outras cardiovasculares. ${ }^{20}$ Neste sentido, destaca-se que a prevalência da variável assistir televisão por mais de três horas foi significativamente elevada na população masculina $(43,1 \%)$ desta pesquisa, comparado ao dado nacional $(28,1 \%)$ e estadual $(27,3 \%)$.

A prática de atividade física também foi uma variável estudada e o resultado demonstrou que as mulheres de Itajaí assistidas pela ESF realizam menos atividade física em seu tempo livre (39\%) quando comparadas às do Brasil $(p<0,001)$ e às de Florianópolis $(p<0,006)$.

Estudo publicado recentemente ${ }^{2}$ revela que no período de 1980 a 2013 o sobrepeso e a obesidade juntos aumentaram 27,5\%, ou seja, de 857 milhões em 1980, para 2,1 bilhões em 2013. Em todo o mundo, a proporção de homens que estavam acima do peso aumentou de 28,8\% em 1980 para 36,9\% 
em 2013, e a proporção de mulheres na mesma condição aumentou de 29,8\% para 38\%. Os aumentos foram registrados nos países desenvolvidos e em desenvolvimento, mas com diferenças entre os sexos.

Nos países desenvolvidos há mais homens do que mulheres com sobrepeso e obesidade, enquanto nos países em desenvolvimento o sobrepeso e a obesidade são mais prevalentes em mulheres do que em homens e essa associação persistiu ao longo do tempo. Em 2013, a prevalência de obesidade foi maior nas mulheres em países desenvolvidos e em desenvolvimento do que nos homens. ${ }^{2}$ Vale salientar que na amostra estudada houve diferenças significativas superiores em relação ao sobrepeso e obesidade para os homens quando comparados às mulheres.

Outro fator de risco para doenças crônicas é o consumo abusivo de álcool. Dados demonstram que as estimativas de dependência de álcool variam de $9 \%$ a $12 \%$ de toda a população adulta, sendo três a cinco vezes maiores em homens em comparação as mulheres. ${ }^{21} \mathrm{Em}$ um estudo populacional, realizado em município de pequeno porte no Paraná, observou que $22,5 \%$ da amostra relatou abuso de bebida alcoólica. ${ }^{22}$ Comparando com este estudo a amostra, o sexo masculino apresentou maior prevalência no consumo excessivo de álcool $(30,6 \%)$.

Aautoavaliação do estado de saúde é um indicador válido e relevante do estado de saúde de indivíduos e de populações. Esse indicador está fortemente relacionado a medidas objetivas de morbidade e de uso de serviços, constituindo-se um preditor poderoso de mortalidade, independentemente de outros fatores. ${ }^{23-25}$

Com relação à autoavaliação negativa do estado de saúde, encontrou-se prevalências muito elevadas em ambos os sexos (19,1\% para homens e 30,9\% para mulheres) diferindo estatisticamente dos dados nacional e estadual e do estudo realizado com beneficiários da saúde complementar em que o resultado foi satisfatório, apenas $3,8 \%$ dos indivíduos estudados consideraram seu estado de saúde como ruim e muito ruim, sendo maior entre as mulheres $(5,2 \%)$ do que entre os homens $(2,1 \%) .{ }^{26}$

Peres et al. ${ }^{27}$ analisaram fatores associados à autoavaliação da saúde em adultos e observaram que, quanto maior o número de morbidades autorreferidas, maior a proporção de indivíduos com autoavaliação de saúde negativa, sendo o efeito das morbidades maior entre as mulheres. Recomenda-se que a autoavaliação de saúde integre o sistema de vigilância à saúde, pois é de obtenção relativamente simples na realização de inquéritos populacionais.

As frequências estimadas de doenças autorreferidas são influenciadas pela cobertura da assistência à saúde local, podendo subestimar, em maior ou menor grau, a prevalência real do fator de risco na população, fornecendo informações úteis para avaliar a demanda por cuidados de saúde originada pela presença do fator. ${ }^{13}$

Dados nacionais representativos referentes a brasileiros de 20 anos de idade ou mais mostram um aumento do diabetes autorrelatado, de 3,3\% em 1998 para 5,3\% em 2008 e para 5,6\% em 2011, sendo $5,2 \%$ no sexo masculino e $6,0 \%$ no sexo feminino. ${ }^{12}$ Conforme os dados atuais do VIGITEL 2013, a diabetes autorreferida foi relatada por $6,5 \%$ dos homens e $7,2 \%$ das mulheres. ${ }^{13} \mathrm{~A}$ dislipidemia foi relatada por $80 \%$ dos homens adultos de uma amostra de 368 indivíduos em um estudo realizado na região Sul do Brasil. ${ }^{28} \mathrm{~A}$ hipercolesterolemia foi relatada por $22 \%$ dos adultos e por um terço dos adultos de 45 anos ou mais, em uma amostra representativa de adultos que moravam em cidades com 100.000 habitantes ou mais. ${ }^{29}$ 


\section{Conclusão}

Os resultados demonstraram que as maiores prevalências de fatores de risco para doenças crônicas não transmissíveis nestes usuários do Sistema Único de Saúde estão relacionados à tríade ingestão alimentar inadequada, inatividade física e tabagismo, salientando as proporções elevadas de autoavaliação negativa da saúde, considerando que este indicador provavelmente está relacionado ao número de morbidades adquiridas. Conhecer a situação real desses fatores para cada região possibilita a adoção de medidas específicas eficazes para promoção da saúde e prevenção das DCNT.

Um fator limitante para este estudo refere-se à comparação realizada entre a amostra e o inquérito nacional promovido pelo VIGITEL. A escolha das unidades de saúde como local principal para a coleta das entrevistas pode ter resultado em amostra de participantes de pesquisa com nível socioeconômico mais baixo que a média do município, enquanto que o inquerito VIGITEL, ao realizar sua amostra por meio da telefonia fixa, pode implicar no contrário, visto que a telefonia fixa tem sido preterida pela população de menor poder aquisitivo. Assim, nas significâncias encontradas para as comparações entre os grupos, a condição socioeconômica pode ter sido uma variavel confundidora, causando uma maximização das probabilidades de diferenças entre o Brasil e Florianópolis e a amostra.

Estudos como este dão suporte científico para a elaboração de projetos na área de saúde, principalmente na assistência básica, na qual se concentra a maioria das consultas e atendimentos, que trarão melhoria da qualidade dos serviços de assistência à saúde e uma redução tão desejada da incidência e dos gastos públicos com DCNT e, consequentemente, melhor qualidade de vida à população.

\section{Referências}

1. Moura EC, Silva AS, Malta DC, Morais Neto OL. Fatores de risco e proteção para doenças crônicas: vigilância por meio de inquérito telefônico, VIGITEL, Brasil, 2007. Cad Saúde Pública. 2011;27(3):486-96. DOI: http://dx.doi.org/10.1590/ S0102-311X2011000300009

2. Ng M, Fleming T, Robinson M, Thomson B, Graetz N, Margono C, et al. Global, regional, and national prevalence of overweight and obesity in children and adults during 1980-2013: a systematic analysis for the Global Burden of Disease Study 2013. Lancet. 2014;384(9945):766-781. DOI: http://dx.doi.org/10.1016/S0140-6736(14)60460-8

3. Yokota RTC, Vasconcelos TF, Ito MK, Dutra ES, Baiochi KC, Merchán-Hamann E, et al. Prevalência de fatores de risco para doenças crônicas não-transmissíveis em duas regiões do Distrito Federal. Com Ciênc Saúde. 2007;18(4):289-96.

4. Brasil. Ministério da Saúde. Plano de ações estratégicas para o enfrentamento das doenças crônicas não transmissíveis (DCNT) no Brasil 2011-2022. Brasília: Ministério da Saúde; 2011.

5. Silva JDT, Müller MC. Uma integração teórica entre psicossomática, stress e doenças crônicas de pele. Estud Psicol. 2007;24(2):247-56. DOI: http://dx.doi.org/10.1590/S0103-166X2007000200011

6. Schmidt MI, Duncan BB, Silva GAS, Menezes AM, Monteiro CA, Barreto SM, et al. Chronic non-communicable diseases in Brazil: burden and current challenges. Lancet. 2011;377(9781):1949-61. DOI: http://dx.doi.org/10.1016/S01406736(11)60135-9

7. Brasil. Instituto Brasileiro de Geografia e Estatística - IBGE. Pesquisa Nacional de Saúde 2013: percepção do estado de saúde, estilo de vida e doenças cronicas. Brasil. Grandes regiões e unidades da federação. Rio de Janeiro: Instituto Brasileiro de Geografia e Estatística; 2014.

8. Gaziano TA, Galea G, Reddy KS. Scaling up interventions for chronic disease prevention: the evidence. Lancet. 2007;370(9603):1939-46. DOI: http://dx.doi.org/10.1016/S0140-6736(07)61697-3

9. Brasil. Ministério da Saúde. O Programa Saúde da Família e a atenção básica no Brasil. Brasília: Ministério da Saúde; 2002. 
10. Silva LS, Cotta RMM, Rosa COB. Estratégias de promoção da saúde e prevenção primária para enfrentamento das doenças crônicas: revisão sistemática. Rev Panam Salud Publica. 2013;34(5):343-50.

11. Instituto Brasileiro de Geografia e Estatística. IBGE. Cidades: dados básicos de 2010 [Internet]; 2010. [citado 2012 Maio 20]. Disponível em: http//www.ibge.gov.br/cidadesat/painel/painel.php?codmun=410840

12. Malta DC, Dimech CPN, Moura L, Silva Junior JB; Grupo Técnico de Monitoramento do Plano de DCNT. Balanço do primeiro ano de implantação do Plano de Ações Estratégicas para o Enfrentamento das Doenças Crônicas Não Transmissíveis (DCNT) no Brasil, 2011 a 2022. Epidemiol Serv Saúde. 2013;22(1):171-8. DOI: http://dx.doi.org/10.5123/ S1679-49742013000100018

13. Brasil. Ministério da Saúde. Vigitel Brasil 2013: Vigilância de fatores de risco e proteção para doenças crônicas por inquérito telefônico. Brasília: Ministério da Saúde;2014. 120 p.

14. World Health Organization. Preventing chronic diseases: a vital investment. Geneva: World Health Organization; 2005.

15. World Health Organization. Overweight and obesity. Geneva: World Health Organization; 2011.

16. Facina T. Pesquisa Especial de Tabagismo (PETab) - Relatório Brasil. Rev Bras Cancerol. 2011;57(3):429-30.

17. Longo GZ, Neves J, Castro TG, Pedroso MRO, Matos IB. Prevalência e distribuição dos fatores de risco para doenças crônicas não transmissíveis entre adultos da cidade de Lages (SC), sul do Brasil, 2007. Rev Bras Epidemiol. 2011;14(4):698708. DOI: http://dx.doi.org/10.1590/S1415-790X2011000400016

18. World Health Organization. Diet, nutrition and the prevention chronic diseases. Geneva: World Health Organization;2003. PMID: 14564933

19. Brasil. Ministério da Saúde. Guia Alimentar para a População Brasileira: Promovendo a alimentação saudável [Internet]. Brasília;2008. [citado 2014 Mar 25]. Disponível em: http://bvsms.saude.gov.br/bvs/publicacoes/guia_alimentar_populacao_ brasileira_2008.pdf

20. Novais M, Leite F. Hábitos de vida - Uma análise da alimentação, do sedentarismo e do tabagismo. Instituto de Estudos de Saúde Complementar [Internet]. São Paulo; 2011. [citado 2014 Mar 28]. Disponível em: http://documents.scribd.com. s3.amazonaws.com/docs/3bf6qpfzuo3oglic.pdf

21. Laranjeira R, Pinsky I, Sanches M, Zaleski M, Caetano R. Alcohol use patterns among Brazilian adults. Rev Bras Psiquiatr. 2010;32(3):231-41. DOI: http://dx.doi.org/10.1590/S1516-44462009005000012

22. Yokota RTC, Iser BPM, Andrade RLM, Santos J, Meiners MMMA, Assis DM, et al. Vigilância de fatores de risco e proteção para doenças e agravos não transmissíveis em município de pequeno porte, Brasil, 2010. Epidemiol Serv Saúde. 2012;21(1):55-68. DOI: http://dx.doi.org/10.5123/S1679-49742012000100006

23. Halford C, Wallman T, Welin L, Rosengren A, Bardel A, Johansson S, et al. Effects of self-rated health on sick leave, disability pension, hospital admissions and mortality. A population-based longitudinal study of nearly 15,000 observations among Swedish women and men. BMC Public Health. 2012;12:1103. DOI: http://dx.doi.org/10.1186/1471-2458-12-1103

24. Franks P, Gold MR, Fiscella K. Sociodemographics, self-rated health, and mortality in the US. Soc Sci Med. 2003;56(12):250514. DOI: http://dx.doi.org/10.1016/S0277-9536(02)00281-2

25. Ilder EL, Benyamini Y. Self-rated health and mortality: a review of twenty-seven community studies. J Health Soc Behav. 1997;38(1):27-37.

26. Malta DC, Oliveira MR, Moura EC, Silva SA, Zouain CS, Santos FP, et al. Fatores de risco e proteção para doenças crônicas não transmissíveis entre beneficiários da saúde suplementar: resultados do inquérito telefônico Vigitel, Brasil, 2008. Ciênc Saúde Coletiva. 2011;16(3):2011-22. DOI: http://dx.doi.org/10.1590/S1413-81232011000300035

27. Peres MA, Masiero GZ, Longo GZ, Rocha GC, Matos IB, Najnie K, et al. Auto-avaliação da saúde em adultos no Sul do Brasil. Rev Saúde Pública. 2010;44(5):901-11. DOI: http://dx.doi.org/10.1590/S0034-89102010000500016

28. Borba E, Philipi A, Nascimento F, Guimarães A, Boff R, Spada P, et al. Perfil lipídico e obesidade em homens de um município da Região Sul do Brasil. Sci Med. 2012;22(1):18-24.

29. Nascimento Neto RM; Sociedade Brasileira de Cardiologia. Atlas Corações do Brasil [Internet]. São Paulo; 2010. [citado 2014 Maio 21]. Disponível em: http://www.prefeitura.sp.gov.br/cidade/secretarias/upload/saude/arquivos/programas/ Atlas_CoracoesBrasil.pdf 
Carine Baumgartel. Curso de Nutrição, Universidade do Vale do Itajaí. Itajaí, SC, Brasil. carine_baumgartel@ hotmail.com

Mihaela Onofrei. Curso de Nutrição, Universidade do Vale do Itajaí. Itajaí, SC, Brasil. iubimic2002@yahoo.com

Leo Lynce Valle Lacerda. Programa de Mestrado Profissional em Saúde e Gestão do Trabalho, Universidade do Vale do Itajaí. Itajaí, SC, Brasil. leolynce@ univali.br (Autor correspondente)

Luciane Peter Grillo. Programa de Mestrado Profissional em Saúde e Gestão do Trabalho, Universidade do Vale do Itajaí. Itajaí, SC, Brasil. grillo@ univali.br

Tatiana Mezadri. Programa de Mestrado Profissional em Saúde e Gestão do Trabalho, Universidade do Vale do Itajaí. Itajaí, SC, Brasil. mezadri@univali.br 